\title{
The Evaluation Value of Emergency and Critical Illness Scoring System for Emergency Medical Patients
}

\author{
XIAOQING XIONG, RONG WANG, C. ZHAO AND BING WANG* \\ Department of Emergency, The Second Clinical College of Yangtze University, Jingzhou 434020, Hubei, China
}

\section{Xiong et al.: Critical Illness Scoring System for Emergency Medical Patients}

\begin{abstract}
To explore the application value of three emergency and critical illness scoring systems in the prognosis assessment of emergency medical patients is the main objective. The 200 emergency medical cases received in the emergency department of our hospital were selected as the research objects and the $28 \mathrm{~d}$ survival of the patients was used as the observation end point. The patients were divided into the survival group 156 cases and the death group 44 cases; statistical analysis of the rapid emergency treatment within $24 \mathrm{~h}$ of admission to the two groups; differences in rapid emergency medicine score, modified early warning score, acute physiology and chronic health evaluation II score, statistical analysis of the general physiological indicators and general data of the two groups of patients within $24 \mathrm{~h}$ of admission; the work of subjects, receiver operating characteristic curve was used to analyze the differences between the three scoring systems in predicting the prognosis of emergency medical patients and the logistic multivariate regression model was used to analyze the relevant factors affecting the prognosis of patients. The rapid emergency medicine score, modified early warning score and acute physiology and chronic health evaluation II score of the death group were higher than those of the survival group and the difference was statistically significant $(p<0.05)$; the rapid emergency medicine score of the death group predicted the $28 \mathrm{~d}$ death of emergency medical patients and the area under the receiver operating characteristic curve was 0.722 . The area under the curve value under the receiver operating characteristic curve for modified early warning score predicting $28 \mathrm{~d}$ death of emergency medical patients was 0.689 and the area under the curve value under receiver operating characteristic curve for predicting $28 \mathrm{~d}$ death of emergency medical patients by acute physiology and chronic health evaluation II score was 0.842 ; the $28 \mathrm{~d}$ prognostic outcome of patients was used as the dependent variable to establish a logistic regression model. Factor analysis showed that the older the patient, circulatory system disease, neurological disease, intensive care unit enrollment, basic disease types $>2$, decreased saturation of peripheral oxygen and unresponsiveness were independent risk factors for $28 \mathrm{~d}$ death in patients admitted to the emergency medical department $(p<0.05)$. For patients admitted to the emergency medical department, rapid emergency medicine score, modified early warning score and acute physiology and chronic health evaluation II score have certain clinical value in predicting the prognosis of patients at $28 \mathrm{~d}$ and the value of acute physiology and chronic health evaluation II score is relatively high. There are many factors affecting the prognosis of patients and they affect the adverse effects of patients in emergency medical department and there are many factors for the prognostic outcome.
\end{abstract}

Key words: Rapid emergency medical scoring, improved early warning scoring system, acute physiology function and chronic health evaluation II score, emergency department

With the increase of critically ill patients in emergency department in China, how to improve the recognition ability of 'critically ill patients' in emergency department and arrange treatment in time has been a clinical concern ${ }^{[1]}$. The traditional intensive care unit (ICU) critical illness scoring system is designed for ICU patients. Limited by objective conditions, this data cannot be obtained timely and accurately ${ }^{[2]}$. Acute physiology and chronic health evaluation II (APACHE II) system consists of three parts: Acute physiology score (APS), age score and chronic health status score, total score 71 points, the higher the score, the more serious the patient's condition ${ }^{[3,4]}$. Whether the disease severity classification system is effective depends on whether it can predict the mortality of patients, studies have shown that APACHE II score can be used to

*Address for correspondence

E-mail: iceking1204@163.com 
evaluate the prognosis of patients with cardiovascular and cerebrovascular diseases and its score is positively correlated with the mortality of patients ${ }^{[5]}$. The rapid emergency medicine score (REMS) is also an emergency critical scoring system. Due to its simple and convenient characteristics, it has been applied in the evaluation of many major diseases ${ }^{[6]}$. Studies have pointed out that the modified early warning score (MEWS) can effectively early warn potential risk patients and significantly reduce the incidence of missed diagnosis in patients with disease changes. At present, it has been widely used in emergency rescue systems in China and abroad ${ }^{[7,8]}$.

The above three indicators can be used to evaluate the prognosis of patients in emergency department of internal medicine. However, studies have found that single study is prone to missed diagnosis and misdiagnosis. The combination of two or two scores may improve the accuracy of diagnosis ${ }^{[9]}$. However, there are few studies on the combination of three indicators for the prognosis evaluation of clinical emergency medical patients and there is no study to compare the three scoring systems. This study will explore the application value of three critical illness scoring systems in the prognosis evaluation of emergency medical patients.

\section{MATERIALS AND METHODS}

\section{Information}

200 cases of emergency internal medicine in emergency department of our hospital were selected as the research objects. The patients were divided into survival group (156 cases) and death group (44 cases) according to the $28 \mathrm{~d}$ survival situation as the observation endpoint. The patients were collected from January 2019 to December 2020.

Inclusion criteria: Patients aged $\geq 18 \mathrm{y}$; emergency admission; emergency classification is I II; hospital stay more than $24 \mathrm{~h}$, the prognosis of patients with clear outcome; the research programme is approved by the Medical Ethics Committee with the informed consent of the family members.

Exclusion criteria: Patients have died at emergency visits; patients family members voluntarily requested to be discharged and transferred to hospital when the patient's condition was not stable; the lack of relevant data cannot be included in statistical analysis.

Survival group, aged from 18 to $77 \mathrm{y}$ old, with an average age of $59.2 \pm 11.7 \mathrm{y}$ old, including 86 males and
70 females. The death group, aged from 22 to $83 \mathrm{y}$, with an average age of $67.2 \pm 10.0 \mathrm{y}$, including 29 males and 15 females.

\section{REMS score, MEWS score, APACHE II score method}

MEWS scoring: MEWS is currently widely used in the UK. The scoring tool includes five physiological parameters, including respiratory rate, heart rate, arterial systolic pressure, consciousness and body temperature. The body temperature parameter was 0-2 points; pulse, systolic blood pressure, respiratory rate and mental reaction parameters were $0-3$ points and the score range was $0-14$ points, the higher the score, the more serious the patient's condition. Patients with 4 and above need to be monitored ${ }^{[10]}$.

APACHE II score: The system consists of three components, namely 12 items of APS, each score is $0 \sim 4$ points, the total score is $0 \sim 60$ points; age score, divided into five grades : $\leq 44$ y ( 0 points), $45-54$ y ( 2 points), $55-64$ y ( 3 points), $65-75$ y (5 points), $\geq 75$ y (6 points); chronic health status score, including past health status, postoperative complications and surgical methods, calculated the total score of the three, the higher the score, the heavier the disease ${ }^{[11]}$.

REMS score includes six parameters: Blood pressure, respiration, pulse, Glasgow Coma Scale (GCS), age, pulse oxygen saturation $\left(\mathrm{SpO}_{2}\right)$, each parameter assignment $0 \sim 6$ points, 16 points and above patients need close monitoring.

\section{Data collection}

The age, gender, admission mode, ICU admission, disease related system, body temperature, blood pressure, heart rate, respiratory rate, blood glucose and consciousness level within $24 \mathrm{~h}$ after admission were collected in the survival group and the death group.

\section{Statistical processing}

SPSS 21.0 was used for data processing software. In this study, the measurement indexes such as REMS score, MEWS score and APACHE II score were tested by normal distribution, which were in line with approximate normal distribution or normal distribution and expressed as $(\overline{\mathrm{x}} \pm \mathrm{s})$. The data were compared by $\mathrm{t}$ test. $\chi^{2}$ test was used to compare the non-grade count data between groups; logistic regression analysis was used for multivariate analysis; test level $\alpha=0.05$. 


\section{RESULTS AND DISCUSSION}

General data comparison; there was no significant difference in gender, smoking and drinking between the death group and the survival group $(p>0.05)$. There were statistically significant differences in age, admission mode, whether to enter ICU, the main diseases and the types of underlying diseases between the death group and the survival group $(\mathrm{p}<0.05)$ (Table 1).

Comparison of physiological indicators; there was no significant difference in body temperature, systolic blood pressure, diastolic blood pressure, HR and blood glucose between the death group and the survival group $(\mathrm{p}>0.05)$. There was significant difference in $\mathrm{SpO}_{2}$ and consciousness level between death group and survival group $(\mathrm{p}<0.05)$ (Table 2$)$.

REMS score, MEWS score, APACHE II score comparison; the scores of REMS, MEWS and APACHE II in the death group were higher than those in the survival group and the difference was statistically significant $(\mathrm{p}<0.05)($ Table 3$)$.

The area under the curve (AUC) value of the REMS score in the death group was 0.722 , that of the MEWS score was 0.689 and that of the APACHE II score was 0.842 (Table 4 and fig. 1).

Logistic regression model was established by taking the $28 \mathrm{~d}$ prognosis outcome of patients as the dependent variable. Multivariate analysis showed that the older the patient, circulatory system diseases, neurological diseases, ICU admission, basic diseases $>2, \mathrm{SpO}_{2}$ reduction and no response to consciousness level were independent risk factors for $28 \mathrm{~d}$ death of patients admitted to emergency internal medicine $(p<0.05)$ (Table 5).

Accurate assessment of critically ill patients in the emergency department can effectively reduce the rate of misdiagnosis and missed diagnosis. However, there

\section{TABLE 1: GENERAL DATA COMPARISON}

\begin{tabular}{|c|c|c|c|c|}
\hline Common data & Death group $(n=44)$ & Survival group $(n=156)$ & $t / x^{2}$ & p \\
\hline Age $(y)$ & $67.2 \pm 10.0$ & $59.2 \pm 11.7$ & 4.510 & 0.000 \\
\hline Sexuality (\%) & & & 1.632 & 0.201 \\
\hline Male & $29(65.91)$ & $86(55.13)$ & & \\
\hline Female & $15(34.09)$ & $70(44.87)$ & & \\
\hline Smoking (\%) & & & 0.901 & 0.342 \\
\hline Yes & $21(47.73)$ & $62(39.74)$ & & \\
\hline No & $23(52.27)$ & $94(60.26)$ & & \\
\hline Drinking $(\%)$ & & & 0.363 & 0.547 \\
\hline Yes & $22(50)$ & $70(44.87)$ & & \\
\hline No & $22(50)$ & $86(55.13)$ & & \\
\hline Hospitalization (\%) & & & 9.352 & 0.009 \\
\hline Walk & $2(4.55)$ & $24(15.38)$ & & \\
\hline Flat bogie wagon & $32(72.73)$ & $74(47.44)$ & & \\
\hline Wheel chair & $10(22.73)$ & $58(37.18)$ & & \\
\hline Whether to enter ICU (\%) & & & 4.394 & 0.036 \\
\hline Yes & $32(72.73)$ & $86(55.13)$ & & \\
\hline No & $12(27.27)$ & $70(44.87)$ & & \\
\hline Major disease (\%) & & & 14.055 & 0.015 \\
\hline Circulating system & $14(31.82)$ & $26(16.67)$ & & \\
\hline Nervous system & $13(29.55)$ & $22(14.1)$ & & \\
\hline Digestive system & $4(9.09)$ & $29(18.59)$ & & \\
\hline Respiratory system & $5(11.36)$ & $35(22.44)$ & & \\
\hline Blood system & $3(6.82)$ & $20(12.82)$ & & \\
\hline Miscellaneous & $5(11.36)$ & $24(15.38)$ & & \\
\hline Basic diseases (\%) & & & 5.023 & 0.025 \\
\hline$\leq 2$ & $12(27.27)$ & $72(46.15)$ & & \\
\hline$>2$ & $32(72.73)$ & $84(53.85)$ & & \\
\hline
\end{tabular}


TABLE 2: COMPARISON OF PHYSIOLOGICAL INDEXES

\begin{tabular}{|c|c|c|c|c|}
\hline Physiologic index & Death group $(n=44)$ & Survival group $(n=156)$ & $t / x^{2}$ & $\mathrm{p}$ \\
\hline Body temperature $\left(^{\circ}\right)$ & $37.08 \pm 0.06$ & $37.10 \pm 0.07$ & -1.880 & 0.062 \\
\hline Systolic pressure (SBP) & $140.8 \pm 12.0$ & $138.5 \pm 10.7$ & 1.149 & 0.252 \\
\hline Diastolic pressure (DBP) & $79.6 \pm 8.5$ & $80.4 \pm 8.1$ & -0.557 & 0.578 \\
\hline Heart rate (HR) (beats/min) & $96.4 \pm 9.0$ & $94.0 \pm 8.5$ & 1.581 & 0.116 \\
\hline Blood sugar (mmol/L) & $6.30 \pm 0.81$ & $6.12 \pm 0.77$ & 1.316 & 0.190 \\
\hline $\mathrm{SpO}_{2}(\%)$ & $96.38 \pm 1.20$ & $98.57 \pm 0.70$ & -11.551 & 0.000 \\
\hline Level of consciousness (\%) & & & 8.155 & 0.043 \\
\hline Consciousness & $2(4.55)$ & $29(18.59)$ & & \\
\hline Response to sound & $9(20.45)$ & $35(22.44)$ & & \\
\hline Response to pain & $10(22.73)$ & $41(26.28)$ & & \\
\hline Adiaphoria & $23(52.27)$ & 51 (32.69) & & \\
\hline
\end{tabular}

TABLE 3: COMPARISON OF REMS SCORE, MEWS SCORE AND APACHE II SCORE ( $\overline{\mathbf{x}} \pm \mathbf{s})$, POINTS

\begin{tabular}{lcccc}
\hline Peer group & $\mathrm{n}$ & REMS score & MEWS score & APACHE II score \\
\hline Death group & 44 & $12.60 \pm 2.80$ & $10.20 \pm 2.64$ & $26.91 \pm 3.02$ \\
Survival group & 156 & $7.46 \pm 1.74$ & $6.60 \pm 1.25$ & $16.05 \pm 2.20$ \\
$\mathrm{t}$ & & 14.921 & 12.748 & 26.487 \\
$\mathrm{p}$ & & 0.000 & 0.000 & 0.000 \\
\hline
\end{tabular}

TABLE 4: THE PROGNOSTIC VALUE OF REMS SCORE, MEWS SCORE AND APACHE II SCORE IN EMERGENCY INTERNAL MEDICINE PATIENTS

\begin{tabular}{lcccccc}
\hline Index & Cutoff value & Sensitivity & Specificity & Omission diagnose rate & Misdiagnosis rate & AUC \\
\hline REMS score & 9.74 & 70.38 & 68.17 & 29.62 & 31.83 & 0.722 \\
MEWS score & 8.68 & 72.46 & 58.47 & 27.54 & 41.53 & 0.689 \\
APACHE II score & 19.84 & 80.61 & 64.29 & 19.39 & 35.71 & 0.842 \\
\hline
\end{tabular}

TABLE 5: LOGISTIC MULTIVARIATE REGRESSION MODEL

\begin{tabular}{lccccccc}
\hline Factor & B & Standard error (SE) & Walds & p & Odds ratio (OR) & 95 \% Confidence interval (Cl) \\
\hline Age & 0.701 & 0.284 & 6.093 & 0.009 & 2.016 & 1.155 & 3.517 \\
Hospitalization & 0.295 & 0.157 & 3.531 & 0.096 & 1.343 & 0.987 & 1.827 \\
This major disease & 0.446 & 0.185 & 5.812 & 0.013 & 1.562 & 1.087 & 2.245 \\
ICU & 0.527 & 0.240 & 4.822 & 0.039 & 1.694 & 1.058 & 2.711 \\
Basic diseases & 0.586 & 0.252 & 5.407 & 0.032 & 1.797 & 1.096 & 2.944 \\
$\mathrm{SpO}_{2}$ & -0.527 & 0.263 & 4.015 & 0.048 & 0.590 & 0.353 & 0.989 \\
Level of consciousness & 0.708 & 0.304 & 5.424 & 0.031 & 2.030 & 1.119 & 3.683 \\
Constant term & 1.395 & 0.779 & 3.207 & 0.116 & 4.035 & 0.876 & 18.575 \\
\hline
\end{tabular}

is no unified evaluation method to predict the prognosis of emergency medical patients. APACHE II score is the most widely used, with the characteristics of accuracy, scientificity, objectivity and credibility ${ }^{[12,13]}$. In this study, we selected relatively easy-to-operate MEWS, REMS and APACHE II scores for comparison and analyzed the advantages and disadvantages of each scoring method.

REMS score has the advantages of simple operation, low cost and convenient access to data, which can be used as the standard for emergency triage. REMS scores only need to measure five physiological indicators and need no high-precision equipment to evaluate.
Studies have shown that the scoring system can, not only assess the risk of pre-hospital transport, but also evaluate the prognosis of patients after admission. The sensitivity and specificity of identifying potential critically ill patients are satisfactory, which can be used as an important means of preliminary prediction of the condition and prognosis of critically ill patients ${ }^{[14,15]}$.

In recent years, due to the characteristics of short time consuming and easy operation of MEWS score, it is increasingly widely used in hospital emergency and emergency system. Previous studies have shown that APACHE II score is used in the occurrence of delirium in patients with severe acute kidney injury and severe 


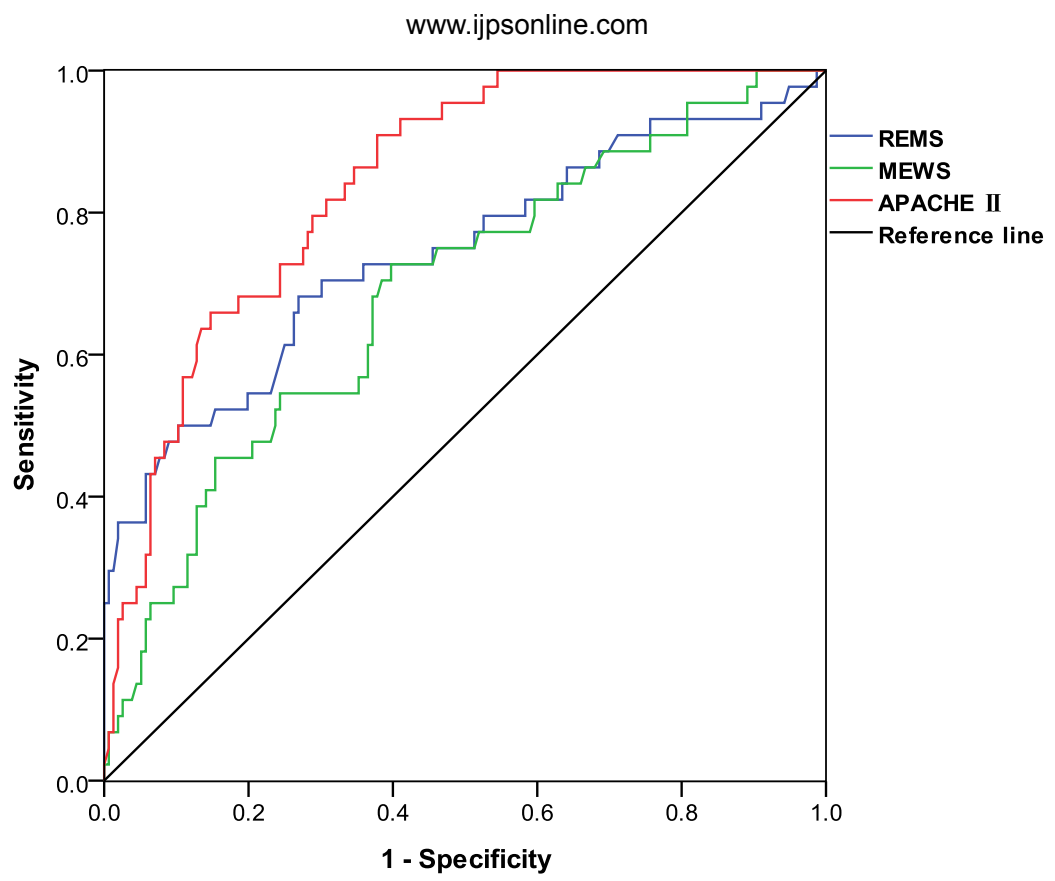

Fig. 1: ROC curve of REMS score, MEWS score and APACHE II score in predicting prognosis of emergency internal medicine patients

pneumonia mechanical ventilation and the prediction effect is good. In addition, APACHE II score is also used to diagnose the severity of other diseases ${ }^{[16,17]}$. Receiver operating characteristic (ROC) value is often used to reflect the predictive value of a certain method for individual prognosis. In this case, the AUC value under the ROC curve of APACHE II score for predicting the $28 \mathrm{~d}$ mortality of emergency medical patients was 0.842 , which was close to 0.85 in Knaus data, indicating that the predictive ability for individuals was also close to the reference level. The results of this study showed that the REMS score, MEWS score and APACHE II score of the death group were higher than those of the survival group and the difference was statistically significant. APACHE-II scoring system is simple and reasonable in design and easy to implement. It can quantitatively calculate the death risk of severe patients and can dynamically score. The maximum difference of subsequent daily parameters is taken to calculate the score. Through the detection and evaluation of indicators under this project item, the physiological disorder of the body can be evaluated, so as to evaluate the severity and prognosis of patients and provide a basis for clinical treatment.

This study showed that the predictive effects of the three scoring systems on the $28 \mathrm{~d}$ mortality of patients from high to low were APACHE II, REMS and MEWS scores. Further studies showed that the logistic regression model was established with the 28 $\mathrm{d}$ prognosis of patients as the dependent variable. After multivariate analysis, the independent risk factors for 28 d death of patients such as older age, circulatory system diseases, nervous system diseases, ICU admission, basic diseases $>2$ and $\mathrm{SpO}_{2}$ reduction were analyzed.

According to the study, when the MEWS score was performed at the beginning, the score may be low. With the aggravation of the patients symptoms, the score will increase ${ }^{[18]}$. Therefore, it is necessary to repeat several scores when using the MEWS scoring system to evaluate the condition and prognosis of emergency medical patients and apply them in combination with the other two systems to improve the accuracy of diagnosis and reduce the mortality of emergency internal medicine patients. However, this study did not carry out the repeatability test due to time limitation. According to the study, REMS was considered as a simple version of APACHE II score. According to GCS score, REMS was easier to perform than APACHE II and was commonly used in non-surgical emergency patients. In addition, APACHE II is helpful to dynamically observe the trend of disease changes and the effect of various treatment measures, which is an important reference for formulating and adjusting medical measures and revising nursing plans ${ }^{[19]}$.

In this study, the clinical value of REMS score, MEWS score and APACHE II score in patients with emergency internal medicine were compared for the first time. It was found that the value of APACHE II score was higher. However, due to the limited number of cases and the characteristics of disease crisis, this study failed to carry out a certain repeated score. There may be 
some deviations in the experimental results and further research and practice are still needed in the future.

In summary, for patients admitted to emergency department of internal medicine, REMS score, MEWS score and APACHE II score have certain clinical value for predicting the prognosis of patients at $28 \mathrm{~d}$ and APACHE II score has high value. There are many factors affecting the prognosis of patients and there are many factors affecting the adverse prognosis of patients admitted to emergency department.

\section{Conflicts of Interest:}

The authors declared no conflict of interest.

\section{REFERENCES}

1. Oostema JA, Chassee T, Baer W, Edberg A, Reeves MJ. Accuracy and Implications of Hemorrhagic Stroke Recognition by Emergency Medical Services. Prehosp Emerg Care 20201(7):1-6.

2. Pong JZ, Koh ZX, Mas'uud Ibnu Samsudin SF, Chong NL, Ong ME. Validation of the mortality in emergency department sepsis (MEDS) scores in a Singaporean cohort. Medicine 2019;98(34):e16962.

3. Rahmatinejad Z, Tohidinezhad F, Reihani H, Rahmatinejad F, Pourmand A, Abu-Hanna A, et al. Prognostic utilization of models based on the APACHE II, APACHE IV and SAPS II scores for predicting in-hospital mortality in emergency department. Am J Emerg Med 2020;38(9):1841-6.

4. Gok RG, Gok A, Bulut M. Assessing prognosis with modified early warning score, rapid emergency medicine score and worthing physiological scoring system in patients admitted to intensive care unit from emergency department. Int Emerg Nurs 2019;43:9-14.

5. Oxman D, Lohr K, Gupta E, Madara J, Len E, Hseih J. Incidence of multidrug resistant infections in emergency department patients with suspected sepsis. Am J Med Sci 2020;360(6):650-5.

6. Bingyu L, Dingyu L, Yinghao S. Comparison of the prognostic value of three rapid scoring methods in severe trauma. J Clin Emerg 2019;20(3):18-21.

7. Baldira J, Ruiz-Rodriguez JC, Wilson DC, Ruiz-Sanmartin A, Cortes A, Chiscano L, et al. Biomarkers and clinical scores to aid the identification of disease severity and intensive care requirement following activation of an in-hospital sepsis code. Ann Intensive Care 2020;10(1):1-11.

8. Feng XF. The guiding value of modified early warning score in pre-examination and trial diagnosis of patients with chest pain in emergency center. J Guiyang Med Coll 2019;44(8):983-6.

9. Chen QX, Wang GS, Zhao T, Gan LL, Yu X. Evaluation and prognostic value of modified early warning score in patients with severe acute pancreatitis complicated with acute lung injury. J Clin Emerg 2019;8(7):513-6.

10. Xie $\mathrm{T}, \mathrm{Lu} \mathrm{J}$, Wei J. Effect of different scoring systems on diagnosis and prognosis of emergency patients with spontaneous non-traumatic cerebral hemorrhage. China Med Guide 2019;16(14)109-12.

11. Yin YP, Tao J, Duan Z. Study on the value of APACHE II score in evaluating the prognosis of emergency critical patients. Contemp Med 2019;25(27):85-7.

12. Liu HY, Feng H. The feasibility and applicability of modified early warning score (MEWS) and rapid emergency internal medicine score (REMS) in the assessment and prognosis analysis of potential critically ill patients in emergency department. J Appl Clin Nurs 2020;5(7):74.

13. Yang YX, Qi WS, Yu D. The value of MEWS, APACHE II score in evaluating the prognosis of sepsis patients with different syndrome ICU. Chin Med Beijing 2019;8(5):418-21.

14. $\mathrm{Hu} \mathrm{H}$, Yao N, Qiu Y. Comparing rapid scoring systems in mortality prediction of critically ill patients with novel coronavirus disease. Acad Emerg Med 2020;27(6):461-8.

15. Crandall M. Rapid emergency medical services response saves lives of persons injured in motor vehicle crashes. JAMA Surg 2019;154(4):293-4.

16. Hansted AK, Moller MH, Moller AM, Vester-Andersen M. APACHE II score validation in emergency abdominal surgery. A post hoc analysis of the In Care trial. Acta Anaesthesiol Scand 2020;64(2):180-7.

17. Shenying W, Hui C. Effect of emergency criticalness index combined with early warning scoring system on stratification and prognostic evaluation of emergency patients. Shanxi Med J 2020;8(4):424-6.

18. Ozluer YE, Avcil M, Ege D, Yasar KS. Emergency department extracorporeal membrane oxygenation may also include noncardiac arrest patients. Turk J Med Sci 2021;51(2):555-61.

19. Liu $\mathrm{XG}, \mathrm{Fu} \mathrm{QH}, \mathrm{Chen} \mathrm{Y}$. C reactive protein, albumin ratio and multi-score system to evaluate the severity and prognosis of sepsis. J Intern Med 2020;26(4):39-41

This is an open access article distributed under the terms of the Creative Commons Attribution-NonCommercial-ShareAlike 3.0 License, which allows others to remix, tweak, and build upon the work non-commercially, as long as the author is credited and the new creations are licensed under the identical terms

This article was originally published in a special issue,
"Evolutionary Strategies in Biomedical Research and
Pharmaceutical Sciences" Indian J Pharm Sci 2021:83(3)
Spl issue;162-167

